\title{
Immunogenicity of the BNT162b2 mRNA Covid-19 vaccine in elderly people over 85 years of age in Greece: the GREVAXIMO study
}

\author{
Konstantina Kontopoulou ${ }^{1}$. Christos Theodore Nakas ${ }^{2,3} \cdot$ Alexandra Ainatzoglou $^{4} \cdot$ Athina Ifantidou ${ }^{1}$. \\ Paschalina Ntotsi ${ }^{5}$. Christos Katsioulis ${ }^{5} \cdot$ Georgios Papazisis ${ }^{4,6}$ (D)
}

Received: 17 August 2021 / Accepted: 30 September 2021 / Published online: 11 October 2021

(c) The Author(s), under exclusive licence to Springer Nature Switzerland AG 2021

\begin{abstract}
Background Elderly people aged over 85 years were among the first groups to receive the BNT162b2 mRNA Covid-19 vaccine in Greece according to the national priority assignment policy.

Aim The aim of this study was to provide useful insight into the antibody generation taking place post-immunization in elderly individuals aged over 85 .

Methods In the first phase of our study, antibody levels were monitored in a total of 400 participants, while our final sample consisted of 297 subjects. Humoral immune responses were recorded in 69.75\% (95\% CI 65.25-74.25) of vaccinees postfirst dose and in 98.99\% (95\% CI 97.85-100) post-second dose.

Results Overall, a remarkable 40-fold change in IgG levels was observed between the two doses. Subjects displaying low antibody levels after the first dose had significantly higher IgG fold changes than vaccinees whose initial antibody levels were high.

Conclusion Taken together, our findings highlighted the high fold change (41.18) recorded in the titers of neutralizing antibodies after the second dose suggesting the need for its timely administration to elderly individuals.
\end{abstract}

Keywords BNT162b2 mRNA Covid-19 vaccine $\cdot$ Immunogenicity $\cdot$ SARS-CoV-2 $~$ Elderly over age $85 \cdot$ Antibodies · Greece

Georgios Papazisis

papazisg@auth.gr

1 Laboratory of Microbiology, “G. Gennimatas” General Hospital, Thessaloniki, Greece

2 Laboratory of Biometry, University of Thessaly, Phytokou Str, 38446 Nea Ionia, Magnesia, Greece

3 University Institute of Clinical Chemistry, Inselspital, Bern University Hospital, University of Bern, Bern, Switzerland

4 Department of Clinical Pharmacology, School of Medicine, Aristotle University of Thessaloniki, Thessaloniki, Greece

5 Committee of Hospital Infections, "G. Gennimatas" General Hospital, Thessaloniki, Greece

6 Clinical Trials Unit, Special Unit for Biomedical Research and Education, School of Medicine, Aristotle University of Thessaloniki, Thessaloniki, Greece

\section{Introduction}

Like in most countries worldwide, immunization against SARS-CoV-2 in Greece took place according to the national priority assignment policy that prioritized population groups at high risk of spreading the infection or developing a severe COVID-19 course, such as healthcare workers and the elderly, respectively. Apart from comorbidities, the most substantial factor compromising the prognosis of infectious diseases among the elderly is immunosenescence, meaning the normal decline in immunocompetence that occurs with aging [1]. Given that the immunization of the elderly against numerous infectious diseases that would otherwise prove life-threatening has been a long-established prophylactic strategy in this age group, anti-SARS-CoV-2 vaccination was expected to be of great benefit in this high-risk population.

In Greece, vaccination of the elderly began with the Pfizer-BioNTech BNT162b2 mRNA Covid-19 vaccine. In parallel with the overall high levels of effectiveness [2, 3] 
and immunogenicity unanimously reported for this vaccine both in phase III [4] and phase IV [5, 6] clinical trials, realworld data on its effectiveness among the elderly have so far been quite positive [7].

Therefore, the aim of this phase IV clinical study was to provide useful insight into the humoral immune response elicited by the first and second doses of the BNT162b2 vaccine in subjects aged over 85 .

\section{Materials and methods}

According to the national priority assignment policy, subjects aged over 85 were among the first groups to receive the Pfizer-BioNTech BNT162b2 mRNA Covid-19 vaccine. A cohort of 400 vaccinees in the 2 vaccination centers of the "G. Gennimatas" General Hospital of Thessaloniki was followed up and had the immunogenic effect of both vaccination doses measured. The two doses were injected in the time span from 08/02/2021 through 24/04/2021.

Three weeks after the administration of each dose, antigen-specific IgG antibodies against SARS-CoV-2 were monitored in participant-derived serum samples, using the SARS-CoV-2 IgG II Quant assay on the ARCHITECT System. Using this automated two-step chemiluminescent microparticle immunoassay, we performed a qualitative and quantitative measurement of $\operatorname{IgG}$ antibodies against the receptor-binding domain (RBD) of the $\mathrm{S} 1$ subunit of the spike protein of SARS-CoV-2.

In addition, a medical history was recorded at baseline based on participants' self-report. Vaccinees were categorized upon history of type 2 diabetes, hypertension, cardiovascular disease (including arrhythmias, heart failure, coronary disease and history of infarction) and reported malignancy. In all subgroups, the geometric mean concentration (GMC) and its 95\% confidence interval were calculated based on the antibody titers measured.

\section{Statistical analysis}

Participants were classified by antibody titers into responders or non-responders according to the seropositivity threshold of $7.1 \mathrm{BAU} / \mathrm{ml}(50 \mathrm{AU} / \mathrm{ml})$ and methods for binomial proportions were used to estimate proportions of responders along with corresponding 95\% confidence intervals. Descriptive statistics included geometric means of concentration (GMC) of RBD-specific IgG (BAU/ml) and the corresponding 95\% confidence intervals $(95 \%$ CI) of each studied group classified by gender, dose (1st or 2 nd) and comorbidity status. Independent samples $t$ tests were used to assess differences in $\log 10 \mathrm{IgG} \mathrm{lev-}$ els between groups. Linear modeling techniques were employed for the assessment of the covariation of continuous measurements (antibody levels and fold changes). Stata 16.1 (Stata Corp. LLC, College Station, TX) was used for data analysis. $p$ values less than 0.05 were considered statistically significant.

\section{Results}

In the initial phase of our study, antibody levels were monitored in a total of 400 participants aged over 85 years that received the first dose of the vaccine. Our final sample consisted of 297 subjects that were immunized with both doses of the vaccine, since 103 vaccinees did not show up for the second antibody measurement 21 days after the second dose.

Twenty-one days after the first dose, 279 out of the 400 participants received a positive outcome (titers $>7.1$ $\mathrm{BAU} / \mathrm{ml}$ ) in the $\mathrm{IgG}$ assay comprising $69.75 \%$ (95\% CI $65.25-74.25$ ) of the total study population. In the remaining 121 participants who received a negative assay outcome, IgG concentration was lower than $7.1 \mathrm{BAU} / \mathrm{ml}$. There was no statistically significant difference due to gender $(p=0.311)$ (Table 1). Our results demonstrated that immunogenicity boosted post-second dose since 294 of participants comprising 98.99\% (95\% CI 97.85-100) of the total study population $(n=297)$ received a positive outcome in the $\operatorname{IgG}$ assay that was conducted 21 days after the second dose. Only three subjects were defined as nonresponders after the 2 nd dose.

Overall, a 40-fold change in IgG levels was observed between the two doses (Table 1); however, values are plateauing for higher titer levels after the 1st dose. Notably, subjects with low antibody levels had significantly higher changes than subjects with initially high antibody levels. This is indicated in Fig. 1, which depicts antibody titers for each subject after the second vs after the first dose. Reference lines were added indicating the limit of $7.1 \mathrm{BAU} / \mathrm{ml}$ on each axis $(\log 10$ scale). The reference lines indicate the three subjects that were deemed as non-responders, remaining below the limit in both doses.

Concerning underlying comorbidities, there was no statistically significant difference in IgG levels upon history of diabetes mellitus in either dose ( 1 st dose, $p=0.275$, 2nd dose, $p=0.567)$. Similar results were obtained for hypertension status after both doses of the vaccine (1st dose, $p=0.353$, 2nd dose, $p=0.402$ ) and history of malignancy (1st dose, $p=0.075,2$ nd dose, $p=0.192$ ). However, there 
Table 1 Geometric means of concentration (GMC) of anti-SARS-CoV-2 S RBD-specific IgG antibodies 14 days post-immunization with the first and second doses of the BNT162b2 vaccine in vaccinees aged $>85$ years, classified by Ab levels and medical history

\begin{tabular}{|c|c|c|c|c|c|c|c|c|}
\hline & $\begin{array}{l}N \text { post-1st dose } \\
\%\end{array}$ & $\begin{array}{l}\text { Post-1st } \\
\text { dose } \\
\text { IgG GMC } \\
\text { (BAU/ml) }\end{array}$ & $95 \% \mathrm{CI}$ & $\begin{array}{l}N \text { post- } \\
2 \text { nd dose } \\
\%\end{array}$ & $\begin{array}{l}\text { Post-2nd } \\
\text { dose } \\
\text { IgG GMC } \\
\text { (BAU/ml) }\end{array}$ & $95 \% \mathrm{CI}$ & $\begin{array}{l}\text { GMC } \\
\text { Fold-change }\end{array}$ & $95 \% \mathrm{CI}$ \\
\hline \multicolumn{9}{|l|}{ Sex } \\
\hline Female & 236 & 17.86 & $(13.48-23.65)$ & 172 & 492.84 & (399.25-608.37) & 40.61 & $(33.23-49.62)$ \\
\hline Male & 164 & 12.39 & $(8.87-17.31)$ & 125 & 416.80 & $(324.66-535.10)$ & 41.97 & $(32.93-53.49)$ \\
\hline$p$ value $(\mathrm{F}$ vs $\mathrm{M})$ & & 0.100 & & & 0.311 & & & \\
\hline \multicolumn{9}{|l|}{ Diabetes } \\
\hline Yes & 87 & 12.24 & $(7.79-19.24)$ & 62 & 416.99 & $(282.20-616.15)$ & 45.10 & $(32.31-62.95)$ \\
\hline No & 311 & 16.36 & (12.79-20.94) & 233 & 467.99 & (391.97-558.75) & 40.08 & $(33.63-47.77)$ \\
\hline$p$ value & & 0.275 & & & 0.567 & & & \\
\hline \multicolumn{9}{|l|}{ Hypertension } \\
\hline Yes & 248 & 16.64 & $(12.71-21.79)$ & 183 & 484.76 & (395.83-593.67) & 38.99 & $(31.86-47.72)$ \\
\hline No & 152 & 13.50 & $(9.42-19.34)$ & 114 & 421.15 & (323.02-549.09) & 44.92 & $(35.41-56.98)$ \\
\hline$p$ value & & 0.353 & & & 0.402 & & & \\
\hline \multicolumn{9}{|l|}{ Cardiovascular disease } \\
\hline Yes & 104 & 9.54 & $(6.21-14.66)$ & 81 & 381.91 & $(274.06-532.21)$ & 50.12 & $(36.62-68.61)$ \\
\hline No & 293 & 18.09 & $(14.10-23.21)$ & 215 & 492.47 & (409.81-591.80) & 38.44 & $(32.22-45.87)$ \\
\hline$p$ value & & 0.011 & & & 0.166 & & & \\
\hline \multicolumn{9}{|l|}{ Malignancy } \\
\hline Yes & 17 & 38.49 & $(9.13-162.33)$ & 15 & 728.08 & $(333.75-1588.30)$ & 35.60 & (13.94-90.90) \\
\hline No & 381 & 14.63 & (11.78-18.18) & 280 & 447.25 & $(379.08-527.67)$ & 41.98 & $(35.94-49.04)$ \\
\hline$p$ value & & 0.075 & & & 0.192 & & & \\
\hline
\end{tabular}

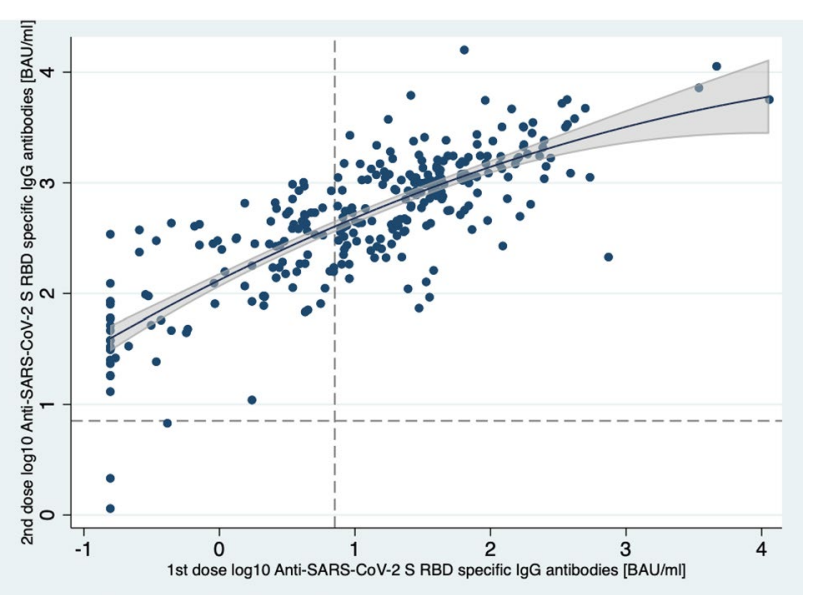

Fig. 1 Antibody titer levels of 1st vs 2nd dose for each participant. Values are plateauing for higher titer levels after 1st dose. Quadratic fit line with corresponding predictive 95\% confidence intervals is shown. Dashed lines correspond to the cutoff value of $7.1 \mathrm{BAU} / \mathrm{ml}$. Only three subjects are indicated as non-responders after the 2nd dose

was a significant difference in IgG levels in subjects presenting with a history of cardiovascular disease compared to those without after the first vaccine dose $(p=0.011)$, which appears to wane after the second dose $(p=0.166)$.

\section{Discussion}

Despite the correlation of aging with a significant reduction in vaccine-induced antibody responses that has been multifold reported $[5,8,9]$, there is still a great need for studies laying focus on the elderly.

In a large-scale matched case-control study on people aged 80-83 years, emergency hospital admissions of elderly vaccinees having received both doses of the BNT162b2 were $75.6 \%$ lower compared to unvaccinated controls, while positive outcomes in SARS-CoV-2 testing were $70.1 \%$ lower in the vaccinated group. These salutary figures emphasize the overall high effectiveness of the BNT162b2 vaccine among the elderly, offering protection against infection and hospitalization [10], while also strongly preventing critical Covid-19 disease and death due to infection [3].

Taking a closer look into the characteristics of the humoral immune response elicited in elderly vaccine recipients, our study highlighted the high fold change (41.18) recorded in the GMC of neutralizing antibodies after the second dose, compared to post-prime dose GMC. Notably, in the 400 recipients of the first dose, mean \% antibody response reached $69.75 \%$, while in the 297 recipients of the second, the respective value was $98.99 \%$. 
An immunogenicity study on 101 elderly recipients of the BNT162b2 first dose showed a geometric mean neutralization titer (GMT) of 46.6, compared to 83.4 in younger subjects, while only $21 \%$ of elderly vaccinees achieved a $50 \%$ virus neutralization capacity, compared to $51 \%$ in younger individuals, respectively [11]. These figures did not change even 12 weeks post-immunization with the first dose, indicating the necessity of second dose administration in elderly with poor neutralization capacity and cautioning against the extension of the interval between the two doses, due to suboptimal protection of the elderly within that time frame. Furthermore, impaired cellular immune responses were also observed in the elderly following the first dose [11]. These findings are well in line with those of another study that monitored antibody responses of elderly vaccinees and found the fold change to be significantly higher in this age group compared to subjects aged under 60 [7]. However, antibody titers still remained lower in the elderly group. Undetectable levels of neutralizing $\operatorname{IgG}$ were reported in $31 \%$ of elderly participants after the second dose, compared to the respective proportion of $2.2 \%$ in individuals aged under 60 . This evidence demonstrates the deficit in the antibody responses elicited in elderly vaccinees due to the inadequate levels of neutralizing antibodies generated [7].

With respect to chronic comorbidities, there has been a negative association of history of high blood pressure and type 2 diabetes with vaccine effectiveness [9, 12], while cardiovascular disease showed no such correlation [9]. In our study, comorbidities did not seem to affect antibody responses, with the exception of vaccinees with cardiovascular disease in whom significantly lower antibody titers were recorded after the first dose, compared to those with a negative history. However, these results regarding comorbidities should be interpreted cautiously since our categorization was based on patients' self-report and not their actual medical registries. The lack of access to the complete medical record of our study participants is a limitation of this study since confounding factors in the apparent association of certain medical conditions with decreased levels of immunogenicity cannot be identified. A further methodological limitation of the present study is the omission of a baseline antibody measurement.

\section{Conclusions}

Taken together, our findings highlighted the high fold change $(F C=41.18)$ recorded in the titers of neutralizing antibodies after the second dose suggesting the need for its timely administration to elderly individuals. Further studies at later time points are needed to longitudinally monitor immune responses of this population, to gain a better understanding of their antibody dynamics and potentially determine their longevity.

Funding This research did not receive any specific grant from funding agencies in the public, commercial, or not-for-profit sectors.

\section{Declarations}

Conflict of interest The authors declare that they have no conflict of interest.

Statement of human and animal rights All methods were carried out in accordance with relevant guidelines and regulations.

Ethical standards The authors certify that they comply with the ethical guidelines for authorship and publishing of the Aging Clinical and Experimental Research Journal.

Ethical approval and informed consent The study protocol was reviewed and approved by the Scientific Committee of "G. Gennimatas" General Hospital (Protocol Number: 2/12.2.2021). All subjects agreed to voluntarily participate in the study and provided written informed consent. The trial is registered at ClinicalTrials.gov (Identifier: NCT04756817)

\section{References}

1. Chen Y, Klein SL, Garibaldi BT et al (2021) Aging in COVID19: vulnerability, immunity and intervention. Ageing Res Rev 65:101-205

2. Dagan N, Barda N, Kepten E et al (2021) BNT162b2 mRNA Covid-19 vaccine in a Nationwide mass vaccination setting. $\mathrm{N}$ Engl J Med 384:142-1423

3. Haas EJ, Angulo FJ, McLaughlin JM et al (2021) Impact and effectiveness of mRNA BNT162b2 vaccine against SARSCoV-2 infections and COVID-19 cases, hospitalisations, and deaths following a nationwide vaccination campaign in Israel: an observational study using national surveillance data. Lancet 397:1819-1829

4. Walsh EE, Frenck RW, Falsey AR et al (2020) Safety and immunogenicity of two RNA-based Covid-19 vaccine candidates. N Engl J Med 383:2439-2450

5. Abu Jabal K, Ben-Amram H, Beiruti K et al (2021) Impact of age, ethnicity, sex and prior infection status on immunogenicity following a single dose of the BNT162b2 mRNA COVID-19 vaccine: real-world evidence from healthcare workers, Israel, December 2020 to January 2021. Eurosurveillance 26:2100096

6. Kontopoulou K, Ainatzoglou A, Nakas C et al (2021) Second dose of the BNT162b2 mRNA vaccine in Greece: the value of timely administration. SSRN Electron J. https://doi.org/10.2139/ ssrn. 3800040

7. Müller L, Andrée M, Moskorz W et al (2021) Age-dependent immune response to the Biontech/Pfizer BNT162b2 COVID-19 vaccination. Clin Infect Dis. https://doi.org/10.1093/cid/ciab381

8. Naaber P, Jürjenson V, Adamson A et al (2021) Antibody response after COVID-19 mRNA vaccination in relation to age, sex, and side effects. MedRxiv. https://doi.org/10.1101/2021.04.19.21255 714

9. Yelin I, Katz R, Herzel E et al (2021) Associations of the BNT162b2 COVID-19 vaccine effectiveness with patient age 
and comorbidities. MedRxiv. https://doi.org/10.1101/2021.03. 16.21253686

10. Mason T, Whitston M, Hodgson J et al (2021) Effects of BNT162b2 mRNA vaccine on Covid-19 infection and hospitalisation among older people: matched case control study for England. MedRxiv. https://doi.org/10.1101/2021.04.19.21255461

11. Collier DA, Ferreira ATM, I, Datir R, Kotagiri P, Lim E, Meng $B$, et al (2021) Age-related heterogeneity in immune responses to SARS-CoV-2 vaccine BNT162b2. MedRxiv. https://doi.org/10. $1101 / 2021.02 .03 .21251054$
12. Chodick G, Tene L, Patalon T et al (2021) The effectiveness of the first dose of BNT162b2 vaccine in reducing SARS-CoV-2 infection 13-24 days after immunization: real-world evidence. MedRxiv. https://doi.org/10.1101/2021.01.27.21250612

Publisher's Note Springer Nature remains neutral with regard to jurisdictional claims in published maps and institutional affiliations. 